\title{
Optimizing the Topological and Combinatorial Complexity of Isosurfaces
}

\author{
Carlos Andújar* $\quad$ Pere Brunet* \\ Antoni Chica* \\ Isabel Navazo* \\ Jarek Rossignac ${ }^{\dagger}$ \\ Àlvar Vinacua*
}

July 7,2004

\begin{abstract}
Since the publication of the original Marching Cubes algorithm, numerous variations have been proposed for guaranteeing water-tight constructions of triangulated approximations of isosurfaces. Most approaches divide the 3D space into cubes that each occupy the space between eight neighboring samples of a regular lattice. The portion of the isosurface inside a cube may be computed independently of what happens in the other cubes, provided that the constructions for each pair of neighboring cubes agree along their common face. The portion of the isosurface associated with a cube may consist of one or more connected components, which we call sheets. The topology and combinatorial complexity of the isosurface is influenced by three types of decisions made during its construction: (1) how to connect the four intersection points on each ambiguous face, (2) how to form interpolating sheets for cubes with more than one loop, and (3) how to triangulate each sheet. To determine topological properties, it is only relevant whether the samples are inside or outside the object, and not their precise value, if there is one. Previously reported techniques make these decisions based on local — per cube - criteria, often using precomputed look-up tables or simple construction rules. Instead, we propose global strategies for optimizing several topological and combinatorial measures of the isosurfaces: triangle count, genus, and number of shells. We describe efficient implementations of these optimizations and the auxiliary data structures developed to support them.
\end{abstract}

Keywords: isosurface extraction, Marching Cubes, Handle Removal, Topological Ambiguity, Triangle Meshes

\section{Introduction}

Let $O$ be a solid object with one or more connected components. A discrete representation of $O$ may be obtained by classifying, against $O$, a set of sample points distributed on the nodes of a regular, axis-aligned three-dimensional grid. Nodes lying inside $O$ or on its boundary are labeled as black and nodes lying outside $O$ are labeled as white. Such a lattice may be constructed in a variety of ways from a polyhedral or curved representation of $O$ through a voxelization process. A similar lattice color-coding may be produced by considering the values of a scalar field at each node. If the value is larger than a prescribed threshold, the node is black; otherwise, it is white.

In many application areas, it is useful to convert the discrete information stored in the black/white coloring of the grid nodes into a continuous boundary model. Most often, this model is a triangle mesh $M$, which approximates the boundary of the original solid object $O$. We say that $M$ is a separating surface for a black/white grid of nodes when it is a manifold triangle mesh that bounds a solid that contains all black nodes and none of the white ones.

Multiple variations of the original Marching Cubes algorithm [9] give a solution to the isosurface extraction problem. However, current techniques are based on local criteria and therefore cannot offer a direct control of topological properties of the extracted mesh, like the genus.

\footnotetext{
*LSI, Software Department and IRI, Institute of Robotics and Informatics. Universitat Politècnica de Catalunya, Barcelona, Spain. \{andujar, pere, isabel, alvar\}@lsi.upc.es, achica@iri.upc.es

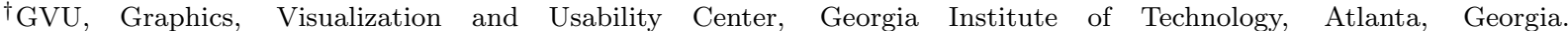
$\{$ jarek@cc.gatech.edu $\}$
} 
Sometimes the connectivity, and hence the topology, of $M$ is unambiguously defined by the in/out classification of the samples and therefore all isosurface extraction algorithms lead to topologically equivalent meshes. But in general, different extraction algorithms may lead to meshes with different topologies. This happens when the isosurface has points that are very close despite being separated by a large geodesical distance over the surface, or when the isosurface contains narrow spikes, as we shall discuss in Sections 2 and 3. The principal focus of prior art in this area was to guarantee that $M$ is a valid boundary of a solid and possibly to guarantee that it is a two-manifold.

In this paper, we propose an approach for selecting amongst all valid topologies the one that minimizes a desired topological or combinatorial cost, which can be the total triangle count, the number of connected shells, or the total genus. Since the topological and combinatorial complexity measures that we strive to minimize are not affected by the placement of the vertices of the isosurface mesh — provided that the mesh still separates the interior samples from the exterior ones - we place each vertex of the isosurface at the midpoints of the lattice edges joining inside and outside samples. When the samples are not binary, or to better define flat regions [1], an application may move these vertices to more appropriate locations along their lattice edge, thus preserving the topological and combinatorial properties of the isosurface that improves other geometric criteria, provided of course that such an adjustment does not produce a self-intersection.

The proposed algorithm is very efficient. It succeeds in optimizing the topological properties of $M$. To ensure topological validity and to provide the application with the freedom of sliding the vertices along the sticks and of adjusting the orientation of each face, we deliberately exclude triangles that lie on a face of a cube.

Our main contributions are:

- The identification and classification of the degrees of freedom in the isosurface extraction algorithms.

- The design of a new isosurface extraction algorithm that guarantees a topologically correct isosurface, by using these degrees of freedom.

- The derivation of two data structures (the X-face graph and the Merge Tree of equivalence classes) that capture the global topological properties of $M$.

- Efficient algorithms, based on a few atomic operations and on the traversal of the X-face graph, for the optimization of the topology of $M$.

- The guarantee that $M$ is two-manifold.

After reviewing the previous work in the next section, we present the degrees of freedom that are implicit in standard Isosurface Extraction algorithms, and propose algorithms and dedicated data structures for isosurface generation with optimization of the topological properties of the final mesh, $M$. The optimization criteria and the corresponding algorithms are presented in Section 7. Finally, we report results on several examples.

\section{Previous Work}

As first noted by Dürst [5], the original Marching Cubes algorithm [9] may produce isosurfaces with holes due to topologically inconsistent decisions on the reconstruction of ambiguous faces, where the borders used by one incident cube do not match the borders of the other incident cube. Several approaches addressing this problem have been published (see [7, 14] for a review).

Disambiguation techniques reported so far have focused on two major concerns: topological consistency, i.e. producing closed surfaces by proper cube polygonalization, and topological correctness, i.e. extracting a surface faithful to the geometry of the real surface.

Consistency can be guaranteed by just considering the inside/outside node classification, regardless of the actual data values. Tetrahedra decomposition techniques $[12,19]$ split each cube into five or six tetrahedra, which always exhibit an unambiguous polygonalization. Preferred polarity methods decide how to slash an ambiguous face of a cell (like the one in Figure $1 \mathrm{c}$ and $\mathrm{d}$ ) using a uniform criterion: always join black nodes or always join white nodes. This decision can be implemented either algorithmically [3] or by using a single-entry lookup table $[8,10]$. All these techniques are generally simple to implement although they do not solve the correctness problem.

Techniques addressing the topological correctness problem infer the proper polygonalization of an ambiguous cube by analyzing its actual data values. As a consequence, these methods are required to provide 
different polygonalization schemes for each ambiguous cube. Most methods only attempt to assure the correctness of the returned surface on the boundary of ambiguous faces. The analysis can be based on face center resampling [16, 18], bilinear interpolation [13] or gradient disambiguation [17]. Only a few methods attempt to recover the original topology also inside the ambiguous cubes either by using critical point analysis [15] or trilinear interpolation $[4,11]$. Note that all these techniques are data-dependent and therefore are noise-sensitive and cannot be applied to binary grids.

All the disambiguation techniques discussed so far are based on local decisions and do not offer any explicit control over the global properties of the extracted surface such as genus, triangle count, or number of shells.

Besides Marching Cubes disambiguation, a number of techniques have been proposed for guaranteeing the topological correctness of the resulting surface. When the desired topology and an approximating shape are known beforehand, one can start with an initial estimate of $M$ and then adjust it to match a given shape by applying topology-preserving operations (see e.g. [2]). Alternatively one may remove topological noise once the surface has been computed, operating directly on the extracted mesh. Yet another approach has also been proposed for removing small handles or tunnels in [6].

\section{Definitions}

The space surrounding the solid $O$ may be decomposed into cubic cells in two different ways. Cubes centered around the nodes are usually referred to as the voxels of a volumetric model. Each voxel inherits the color of the node located at its center. The union of the black voxels may be used as a coarse approximation of $O$. In contrast to voxels, the cubes considered here span the interstice between 8 nodes of the lattice, which are its corners. A cube has 12 edges. Some of them may join white and black nodes and these contain the vertices of the mesh $V=\left\{V_{i}\right\}$.

\subsection{Sheets and Border Edges}

The intersection between the triangle mesh $M$ and the boundary of a given cube forms one or several polygonal cycles. Like most isosurface generation schemes, we require that the edges of these intersection curves form a subset of the edges of $M$. This assumption is fundamental to the Marching Cubes algorithm and to most of its variations, because it ensures that each triangle of $M$ is contained in a single cube. Consequently, the triangles of $M$ may be generated by considering one cube at a time.

The set of triangles of $M$ that lie in a given cube may be empty (when all corners of the cube have the same color) or may form one or more connected components called sheets. Each one of these components is a 2-manifold with boundary. It is bounded by one or more simple cycles of border edges (contained in the faces of the cube). Let $C_{L}$ and $C_{R}$ be the two face-connected cubes sharing a common face $f_{L R}$. To ensure that $M$ is a manifold without boundary, the border edges of the portion of $M$ in the cube $C_{L}$ must match the border edges of the portion of $M$ in the neighbor cube $C_{R}$. This way, each triangle of $M$ has one neighbor across each one if its edges. This requirement has led to several publications that disambiguate the MC algorithm, as discussed in Section 2.

\subsection{X-faces, Loops and X-cubes}

When the four corners of a face have the same color, the face contains no edges of $M$. If when traversing the edges of a face $f$ we see only a transition from inside to outside and one from outside to inside (see Figure 1 $\mathrm{a}, \mathrm{b}$ ), it contains two mesh-vertices and a single border edge of $M$, joining them. This edge will be used as a border edge by the two cubes incident upon $f$.

Finally, when a face $f$ has alternating black and white corners, and hence four mesh-vertices in its boundary, it contains two border edges of $M$. Note that we have a choice in selecting these two edges (Figure $1 \mathrm{c}$ and $\mathrm{d}$ ). We use the term $X$-face to refer to such ambiguous faces.

Once the edges of $M$ have been defined for all the faces, the corresponding borders for any given cube may always be uniquely chained into cycles, which we call loops. These loops are the boundary of the portion of $M$ associated with the cube. We can have at most four loops inside a cube (Figure 2).

$X$-cubes are defined as cubes having no X-faces but having more than one loop. The only MC configuration leading to an X-cube is the one depicted in Figure 2-left. The loops in an X-cube can be connected or not. $\mathrm{X}$-cubes represent quasi-non-manifold parts of $O$ that are not producing $\mathrm{X}$-faces. 


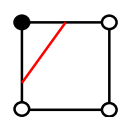

a)

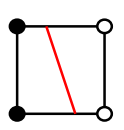

b)

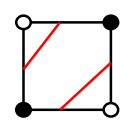

c)

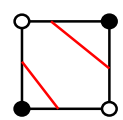

d)

Figure 1: Faces with two vertices ( $\mathrm{a}, \mathrm{b})$ generating a single border edge of $M$. In (c, d), faces generating two border edges of the triangular mesh $M$.
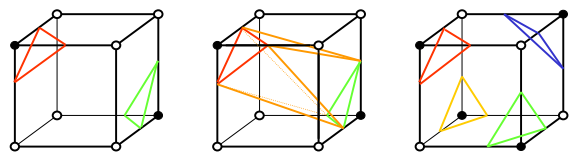

Figure 2: Configurations with two and with four loops. With two loops (left and center), we have two possibilities: two sheets, each homeomorphic to a disk, or a single sheet homeomorphic to an annulus (tunnel or handle).

\section{Degrees of Freedom in isosurface Extraction}

In the previous section we have seen that there are two kinds of cubes inside which the isosurface topology is not uniquely determined: those having $\mathrm{X}$-faces and the $\mathrm{X}$-cubes. This gives us two ways of controlling the topology and the connectivity of the final mesh: we must decide how to slash $\mathrm{X}$-faces and we must decide (in each X-cube) whether to have a different sheet per loop or to connect the two loops. Decisions on X-cubes are obviously independent from decisions on X-faces, as X-cubes (and therefore their neighbors) have no $\mathrm{X}$-faces. Deciding how to slash X-faces and deciding if loops must be connected or not in X-cubes, gives us a number of degrees of freedom that can be used to optimize the topological properties of the final mesh $M$.

Moreover, after having decided which way to slash each X-face and which way we connect loops in each $\mathrm{X}$-cube, individual sheets must be triangulated. Although the available choices may impact the total area of $M$, they do not affect - in our approach - the topology, and hence they are not relevant for the optimization of the number of shells, or the genus. In short,

- Once the choice for all of the X-faces is made, the border edges and hence the loops of the final mesh are completely determined.

- The choice on having separate sheets or connecting loops in individual X-cubes, affects the total number of triangles in the mesh and the topology of the mesh (Figure 2).

- The decision on how to select a triangulation for each sheet among the valid ones has no impact on the topology of the mesh, but it does have an effect on the total surface area of the mesh. Therefore we will not discuss these choices in the present paper.

\section{Tools for a Global Approach}

The objective of the rest of the paper is to propose several algorithms that use the degrees of freedom shown in the last section in order to optimize the topological properties of the final mesh. In this section we will first show that this is not possible with only local decisions, and next we will introduce two efficient data structures (representing global information) for the topological optimization in Marching Cubes.

The Euler-Poincaré formula for a closed manifold triangle mesh $M$ without borders consisting of $V$ vertices, $S$ shells (connected components of $M$ ), and $H$ handles indicates that the total number $T$ of triangles is

$$
T=2 V+4(H-S)
$$

The total number of loops over all cubes will be noted as $L$, while the total number of half border edges over all the faces of the cubes will be noted as $B$. For a particular cube $C$, its number of loops and border edges will be noted as $L_{c}$ and $B_{c}$. Note that $L$ is the sum of all $L_{c}$ and that $B$ equals the sum of all $B_{c}$.

Each vertex of $M$ is bounding eight border edges because it lies on an edge of the grid which has four incident square faces of the adjacent cubes. Each face $f$ contains two coincident border edges, one per cube 
incident upon $f$. Furthermore, each border edge is bounded by two vertices. Hence, the total number of border edges in all cubes is constant:

$$
B=4 V
$$

We also have a relationship among the number of loops $L$, the number of triangles $T$ and the total number of sheets (denoted by $s$ ) inside all cubes. The relation is given by the formula:

$$
T=4 V+2 L-4 s
$$

To prove this relationship, let us first look at a single cube $C$, and let us first assume that we keep the loops separated without connecting them. Then, for a loop having $b$ border edges we generate $t=b-2$ triangles of $M$. If $C$ has $b$ border edges and $l$ loops, we can sum the number of triangles for each of the loops and obtain $t=b-2 l$ as the total number of triangles generated for $C$. By summing this expression for all cubes and taking into account Equation 2 and that $s=L$ (since we do not have connected loops) we can write: $T=B-2 L=4 V-2 L=4 V-2 L-4(s-L)=4 V+2 L-4 s$, which is the above equation. To prove it in the general case where we connect some of the loops, we can simply observe that the above equation is invariant under the connecting loops operation: for every connection between two loops in any of the cubes, $s$ decreases in one while $T$ increases by four (Figure 2).

Also observe that once $\mathrm{X}$-faces have been fixed, since $V$ is constant, $L$ is also constant and we can conclude that $T$ and $s$ are always varying in opposite ways: $s$ decreases as $T$ increases, and vice-versa.

Given that $V$ is fixed, the only available variables for our optimization are $T, H$ and $S$. The previous argument shows that through $s$ we can locally control $T$, and hence (because of Equation 1) $H-S$. Unfortunately, we do not have local control on $H$ and $S$ separately, since they depend on the global structure of the mesh. Figure 3 shows an example in two dimensions where the decisions taken at $A$ and $B$ are coupled in determining the topology of the result (one simply-connected component, two connected components or one component, not simply connected).

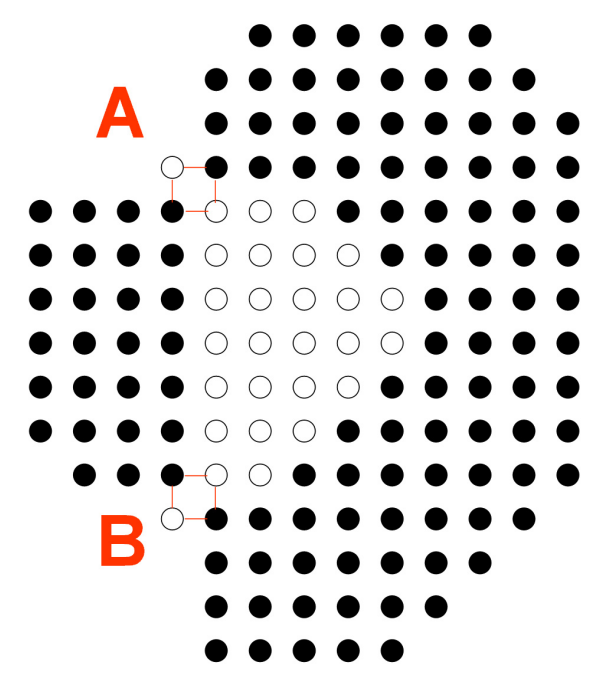

Figure 3: A quasi-non-manifold example in two dimensions, with two X-faces $A$ and $B$. This example shows how the topology cannot be decided by purely local decisions.

In the rest of this section we will present the two new data structures that supply the necessary global information to the mesh generation algorithm: the X-face propagation graph and the merge tree of equivalence classes of vertices.

\subsection{X-face propagation graph}

The $X$-face propagation graph is a convenient tool for deciding on $\mathrm{X}$-face slashing. Consider the abstract graph $G=(\mathcal{V}, E)$ where cubes with at least one X-face correspond to graph nodes and where the X-faces correspond to links between the graph nodes that represent their incident cubes. For this graph to represent a possible choice of how to slash the $\mathrm{X}$-faces, each graph edge is assigned a binary value indicating its slashing choice. 


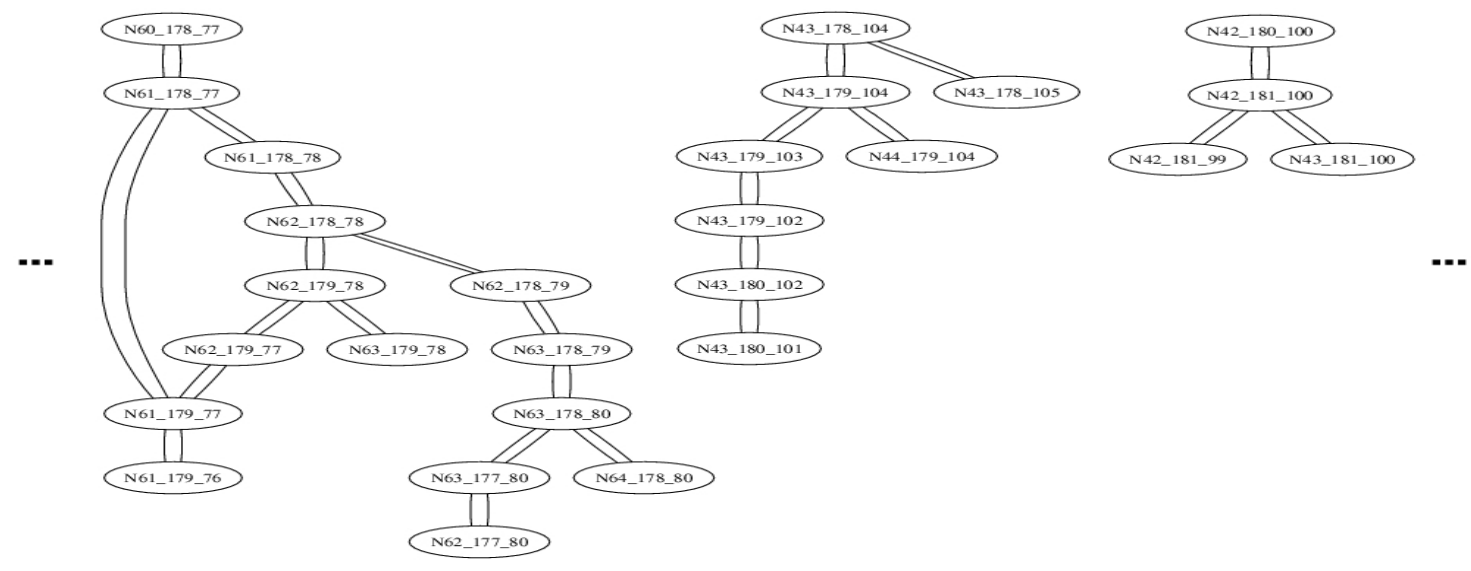

Figure 4: Main components of the X-face graph on the test model.

The X-face graph can be constructed in linear time by a single traversal of the volumetric model. During the traversal, a graph node with label $(i, j, k)$ is inserted into $\mathcal{V}$ if the cube $(i, j, k)$ has at least one $\mathrm{X}$-face. An edge connecting node $(i, j, k)$ with any of its three face-neighbors along directions $\{X+, Y+, Z+\}$ is inserted into $\mathrm{E}$ if the shared face is an $\mathrm{X}$-face. Since this graph is not oriented, only three faces of each cube are considered, so as to avoid edge repetition.

A simple examination of the 256 cube configurations reveals that cubes can have $0,1,2,3$ or 6 X-faces (frequencies over the 256 configurations are shown in Table 1). Since the degree of the graph vertices is bounded by 6 , X-face graphs are sparse, with a small number of edges connecting nodes and only a few high-order nodes.

\begin{tabular}{ccc} 
\# X-faces & \# Configurations & Percentage \\
\hline 0 & 135 & $52.7 \%$ \\
1 & 72 & $28.1 \%$ \\
2 & 30 & $11.7 \%$ \\
3 & 16 & $6.2 \%$ \\
4 & 0 & $0.0 \%$ \\
5 & 0 & $0.0 \%$ \\
6 & 2 & $0.7 \%$
\end{tabular}

Table 1: Frequencies of X-faces over the 256 cube configurations. Note that cubes with 4 or 5 X-faces do not exist.

\begin{tabular}{llrrrrrrrr} 
Dataset & Resolution & Non-empty cubes & Deg 1 & Deg 2 & Deg 3 & Deg 6 & \# arcs & \# cycles & \# components \\
\hline Ship (Fig 6) & $128 \times 128 \times 128$ & 35,020 & 54 & 21 & 0 & 0 & 48 & 0 & 27 \\
Random 1 & $8 \times 8 \times 8$ & 312 & 49 & 9 & 9 & 2 & 53 & 0 & 16 \\
Random 2 & $8 \times 8 \times 8$ & 299 & 34 & 12 & 8 & 2 & 47 & 8 & 13
\end{tabular}

Table 2: Properties of the X-face graph on the test datasets. Deg $n$ stands for the number of graph nodes with degree $n$.

Table 2 shows the number of vertices, edges, components and cycles of the X-face graph on a ship engine's room model (Figure 6) and on two random datasets. Note that on all test models the X-face graph has many connected components and few high-order nodes. Our experiments show that graph cycles might appear, although very rarely in practice. Hence, most of the connected components of the X-face graph are trees.

\subsection{Connectivity merge tree}

The second data structure is related with the equivalence classes of vertices. These equivalence classes initially encode clusters of vertices connected by border edges that are not contained in X-faces (obviously, internal edges of the cube triangulations do not affect these clusters). In other words, two vertices initially 
belong to the same class if and only if they will belong to the same shell regardless of the X-face and X-cube decisions. We will use this additional tool to decide on the individual effect on $H$ and $S$ of a certain choice on the connection of loops of an X-cube or the slashing of an $\mathrm{X}$ face, and we will update the equivalence classes at each decision.

The interest of this data structure lies in the fact that the number of shells $S$ depends on the global connectivity of the mesh, and cannot be determined locally: if a particular slashing choice in a certain Xface connects vertices that were not previously in the same cluster (equivalence class), we are decreasing the total number of shells $S$. At each point in the execution of the algorithm, this set of classes gives a strict upper bound on the number of resulting shells (given the decisions thus far taken), as all vertices in a single equivalence class must lie on the same shell. At the end, when all decisions have been taken, the number of clusters in this data structure is exactly the number $S$ of shells.

We implement this with a merge tree of the vertices $V_{i}$ that is initially constructed in a one-sweep process. In this way we store equivalence classes of vertices, modulo the equivalence relation given by the connectivity along a series of border edges that do not belong to an X-face. That is: initially, two vertices $V_{i}$ and $V_{j}$ are in the same class if there exists a sequence of vertices $V_{i}=V_{0}, V_{1}, \ldots V_{n}=V_{j}$ such that for $k=0 \ldots n-1$, the segment $V_{i} V_{i+1}$ is a border edge that does not belong to an X-face. Notice that these border edges will always remain in the final triangulation.

Using standard data structures we can construct this set of equivalence classes in a single pass of the model, merging classes as we visit the boundary edges of non-X-faces. Finding the canonical representative of a class has a cost of $O\left(\log ^{\star} n\right)$ where $n$ is the number of vertices in the class. Merging two classes can be done in constant time. Therefore the whole data structure is initialized in time bounded by $O\left(n \log ^{\star} n+m\right)$, where $n$ is the total number of vertices in the model and $m$ is the number of voxels.

Furthermore, this data structure supports the dynamic computation of the impact of any choice on any given $\mathrm{X}$-face or $\mathrm{X}$-cube. If the end vertices of the chosen border edges on an X-face or on the two loops of an X-cube belong to the same class, the choice does not affect the number of shells. If however they belong to different classes, then the choice of connecting the classes will reduce in one unit the maximum number of shells attainable.

\section{The Topological Optimization Algorithm}

Our algorithm consists of four main steps, and optimizes the mesh topology by traversing the X-face graph while taking some atomic decisions on how to slash the individual X-faces and on whether to connect the loops inside X-cubes or not:

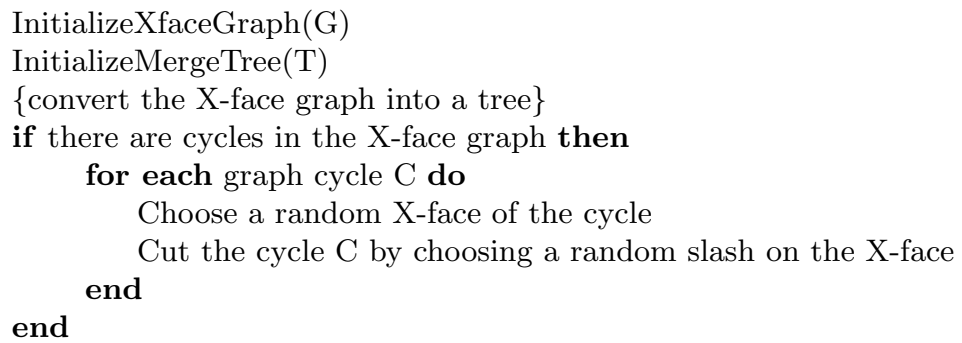




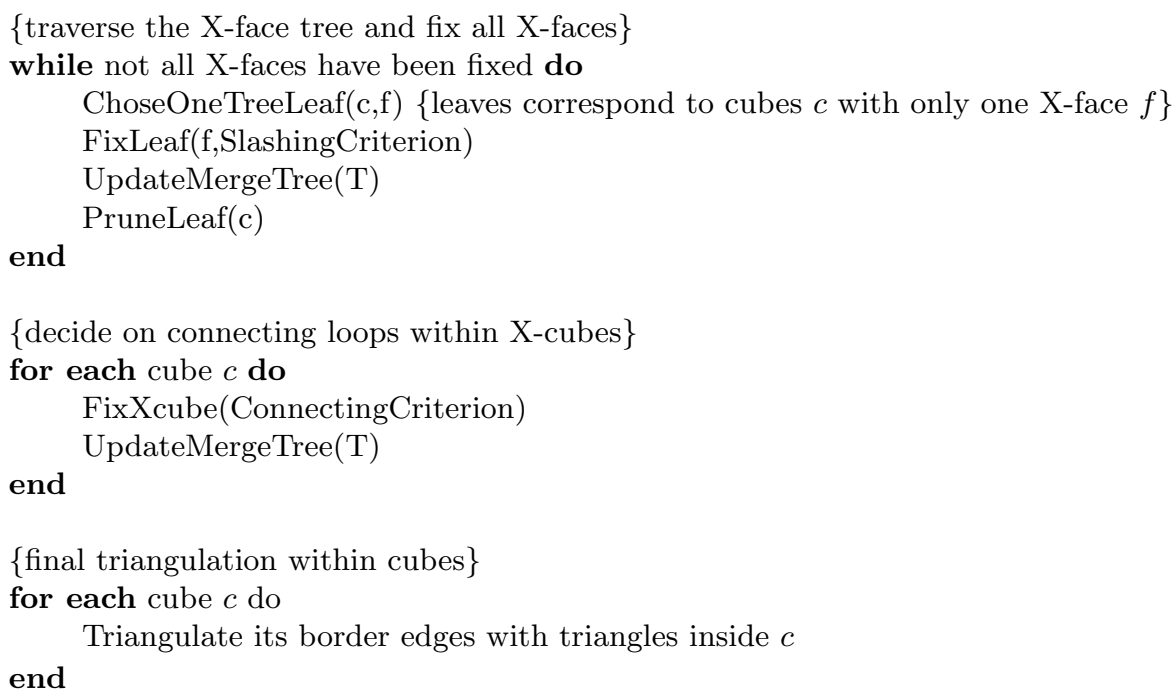

\subsection{Slashing criteria on an X-face $f$}

Observe that the particular choice on how X-faces are slashed affects the total number of loops in the mesh. Switching an X-face $f$ from one slashing choice to the other will always change by exactly one the number of loops in each one of the two cubes adjacent to $f$ (if, before the slash, the two borders of $f$ were part of the same loop in one of the cubes, the slash will split that loop and hence increase the number of loops for that cube; if, before the slash, the two borders of $\mathrm{f}$ were part of two different loops of the cube, the slash will merge these two loops and decrease by one the loops count for this cube). Therefore, depending on the situation, an X-face slash may either leave $L$ unchanged - when the loop count was increased in one of the cubes and decreased in the other one - or increase or decrease it by 2 .

We propose the following four possible criteria to decide which way to slash an X-face (the last two are supported by the Merge Tree encoding the equivalence classes of vertices):

Criterion 1 Take the option that maximizes $L_{c}$ in every cube $c$ sharing the face $f$. We have seen that if $L_{c}$ increases by one, the loop count in the neighbor cube cannot decrease by more than one. Then, the total count $L$ can never decrease. This is used in our greedy algorithm for maximizing $L$.

Criterion 2 Minimize $L_{c}$ in every cube $c$ sharing the face $f$ For the same reasons as in (1), the algorithm will tend to minimize $L$.

Criterion 3 Take the option that does not decrease the number of equivalence classes (if one of the possible choices does so). The algorithm will tend to maximize $S$, as the final number of equivalence classes equals $S$.

Criterion 4 Take the option that decreases the number of equivalence classes (if one of the possible choices decreases it). The algorithm will tend to minimize $S$ (the final number of equivalence classes equals $S)$.

Notice that once the loops in cubes with X-faces have been set, they cannot be joined by triangulations that do not include additional vertices or triangles coplanar with a cube's face. For this reason, we choose to assign a single sheet to each loop obtained by applying these criteria. If however one were interested in these solutions, despite the additional triangles, the decision to connect them or not could be based on criteria like those discussed next for the X-cubes.

\subsection{Criteria on how to connect the loops within X-cubes}

After having fixed the X-faces of the model, we must decide how to connect the remaining free loops (the loops in X-cubes). We must first observe that, when we connect two loops, we have a net increase of $T$ in four (due to Equation 3). Therefore, and due to $T=2 V+4(H-S)$, we have a net increase of $(H-S)$ in one. Taking into account this property, we have the following four options: 
Criterion a Never connect loops. In this case, $(H-S)$ is decreased. This decision tends to generate many small blobs - disconnected shells - . In case of noisy models, irrelevant small features can be easily identified and removed.

Criterion b Always connect loops. Now, $(H-S)$ is increased and either $S$ is decreased or $H$ is increased. At the end we will have a lower number of equivalence classes and a small $S$.

Criterion c Two loops are connected when they belong to the same equivalence class. In this case, $S$ remains constant. But, as we have an increase in $H-S$, the final consequence is an increase of $H$ by one.

Criterion $\mathbf{d}$ Two loops are connected when they do not belong to the same equivalence class. In this case, $S$ is reduced by one. As we have an increase in $H-S$ of also one, $H$ remains constant.

\section{$7 \quad$ Combined decisions}

We have sixteen possible combined decisions that can be taken during the traversal of the X-face graph and the visit of the X-cubes (from 1-a to 4 -d). In what follows, the notation $\min (W)$ stands for the subset $\sigma$ of all meshes $M$ such that $W(m)$ has its minimum value for all $m$ in $\sigma$. The same applies to $\max (W)$. We use the notations High and Low in those cases where we cannot guarantee a maximum or a minimum.

We present here four combinations of these criteria that optimize different aspects of the resulting mesh. They correspond to choices 1-a, 2-b, 3-c and 4-d. In these cases, both atomic decisions are in consonance:

- Minimize $T$, with minimal $H-S, \boldsymbol{L o w}(H)$ and $\boldsymbol{H i g h}(S)$ In case 1-a we maximize $L$ and, since we do not connect loops, we have $s=L$. Then, $T=4 V+2 L-4 s=4 V-2 L$ and the maximization of $L$ leads to $\min (T)$. On the other hand, as $T=2 V+4(H-S)$, we will have $\min (H-S)$, with $\operatorname{Low}(H)$ and $\operatorname{High}(S)$.

- Maximize $T$, with maximal $H-S, \boldsymbol{H} \boldsymbol{i g h}(H)$ and a $\boldsymbol{L o w}(S)$ In case 2-b, $L$ is minimized. Since $s \leq L$, we have that $4(H-S)=2 V+2 L-4 s \geq 2 V-2 L$. The consequence is a $H i g h(H-S)$ with a $\operatorname{High}(H)$ and a $\operatorname{Low}(S)$ (since lowering $L$ and connecting the loops of the X-cubes decreases $S$ ).

- Maximize $S$, with $\boldsymbol{H i g h}(H)$ In case 3-c, the number of shells is maximized, since no decision is taken that reduces the number of shells if it is at all possible. However, criterion c connects again parts of the boundary already in the same component. Since this does not change $S$, but it increases $T$, it must increase $H$ as well. The consequence is we also have a $\operatorname{High}(H)$.

- Quasi-minimize $S$ with $\operatorname{Low}(H)$ Case 4-d tends to $\min (S)$ with $\operatorname{Low}(H)$ since we always connect disconnected equivalence classes. This strategy may not achieve the minimum because ties are resolved randomly when found. It may happen that in one of those cases (where either slashing of the Xface yields the same number of equivalence classes) the decision taken connects vertices that can be connected elsewhere, while failing to connect vertices that could only be connected at that X-face. The event is obviously rare, but can happen (see Table 3).

These four algorithms have been implemented, and their results will be discussed in the next section. The Max/Min global optimal values are always reached provided that the X-face graph has no cycles. In the next section we will see that this is the case in most practical cases.

Our current implementation triangulates each sheet so that no triangle lies on the faces of the cubes. These faces would produce undesirable artifacts when actual data values are used for interpolating the vertex position along the grid edges. It must also be noted that when using criteria 2 and 3 , in order to represent the intersection of two (or more) tunnels, a few cases require the introduction of internal vertices [4].

The remaining twelve combined decisions (1-b, 1-c, .. , 4-c) take conflicting decisions on the X-faces and the X-cube loops, their application being less clear. They will be investigated as part of our future work.

On the other hand, tie situations might occur when the two slashing choices for an X-face fulfill a given criterion. This is often a consequence of the fact that the mesh having the optimal values for $T, H$ or $S$ is not unique. A simple random selection can be used to solve the tie whenever we only target a single magnitude. If this is the case, the output mesh will be a random choice from the set of meshes having the optimal value of the target. A much more interesting approach for solving slashing ties consists in using an ordered pair of combined criteria. This fact enables us to optimize a magnitude while trying to keep small another one. Another approach, is to analyze the two candidate paths in parallel. This is feasible because of the scarce number of cases of this kind that usually arise in practice. 


\section{Examples and discussion}

Figure 5 shows the results of our algorithm with strategies 1-a, 2-b, and 4-d. In this example 3-c yields the same result as 1-a, and is not shown. The test model consists of the edges of a cube plus all of its diagonals. The model has a resolution of $16 \times 16 \times 16$; of the 4096 cells, only 240 have X-faces (but only one per cube), and there are a total of $40 \mathrm{X}$-cubes. The edges of the cube are thicker, so they are stable throughout. The
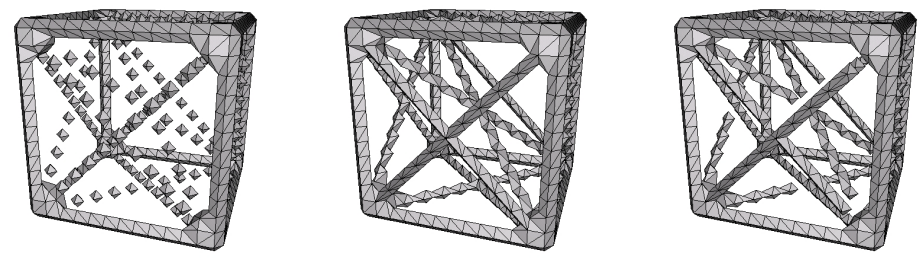

Figure 5: Results of strategies 1-a, 2-b and 4-d on the test cube

diagonals, instead, are thinner, and result in very different reconstructions. In agreement with the expected behavior, we get the triangle, shell and hole-counts summarized in the leftmost block of table 3 One can

\begin{tabular}{r|rrrrrr|rrr} 
& $\mathrm{T}$ & $\mathrm{S}$ & $\mathrm{H}$ & \multicolumn{1}{c}{$\mathrm{T}$} & \multicolumn{1}{c}{$\mathrm{S}$} & \multicolumn{1}{c}{$\mathrm{H}$} & $\mathrm{T}$ & $\mathrm{S}$ & $\mathrm{H}$ \\
\hline 1-a & 2296 & 136 & 5 & 575,776 & 1,182 & 223 & 95,644 & 70 & 58 \\
2-b & 2936 & 1 & 30 & 583,932 & 339 & 1,419 & 97,868 & 9 & 553 \\
3-c & 2296 & 136 & 5 & 577,232 & 1,185 & 590 & 96,856 & 70 & 361 \\
4-d & 2836 & 1 & 5 & 580,076 & 340 & 456 & 96,352 & 9 & 174
\end{tabular}

Table 3: Number of Triangles, Sheets and Holes in the resulting mesh for the test cube (left, all meshes have 1410 vertices), the model in Figure 6 (middle, all meshes have 289,806 vertices) and the skull in Figure 7 (right, all meshes have 47,846 vertices).

observe how 1-a and 3-c tend to minimize the number of triangles, while 2-b and 4-d tend to minimize the number of shells, but 2-b maximizes genus, whereas 4-d minimizes it. Although this example is academic, it displays well the behavior of the different variants of the algorithm.

For a more realistic, albeit less pedagogical example, Figure 6 shows a portion of a ship's engine room, sampled at a resolution of $256 \times 256 \times 256$. The images at the right of Figure 6 are enlarged views of a not-so crowded area (highlighted in blue in the leftmost figure), where differences in the results of strategies 2-b and 3-c are readily seen. The models have been rendered here with all vertices fixed at the midpoints of their cell-edges, which accounts for the irregular appearance, but displays clearly the topology of the result. The processing of this model on a Pentium- 4 running at $1.7 \mathrm{GHz}$ with $256 \mathrm{Mb}$ of ram took: $0.98 \mathrm{~s}$ to build the X-faces graph, $4.23 \mathrm{~s}$ to build the connectivity merge-tree, and $0.41 \mathrm{~s}$ to solve the graph, for a total running-time overhead of $5.62 \mathrm{~s}$ (above the time necessary for the ordinary marching cubes). There are a total of 286,631 non-empty voxels, with 5,739 X-faces; 4,492 cells have only one X-face, 854 have two X-faces, 392 have three X-faces and one has six X-faces. The X-cubes total 289. The merge tree initially has 2,358 components.

Finally, Figure 7 shows the result of applying our algorithm to CT scan data. The model consists of $128 \times 128 \times 128$ voxels. The semi-transparent figure shows the $\mathrm{X}$-face graph of this model, with the degree of the vertices encoded by their color. Red corresponds to cubes with just one X-face (a total of 807), blue to cubes with exactly two X-faces (207), and green for cubes with three X-faces (37). There are no cubes with six X-faces in this model. The figure also shows the results of reconstructing the isosurface with the four different strategies. The processing of this model took, on the same machine, a total of 0.782 seconds, of which 0.117 seconds were spent on building the X-graph, and 0.584 seconds building the merge tree.

Table 3 summarizes the effect on the topology of the different variants of our algorithm on the three models discussed here. Notice how these numbers are in agreement with the properties enumerated in Section 7. 

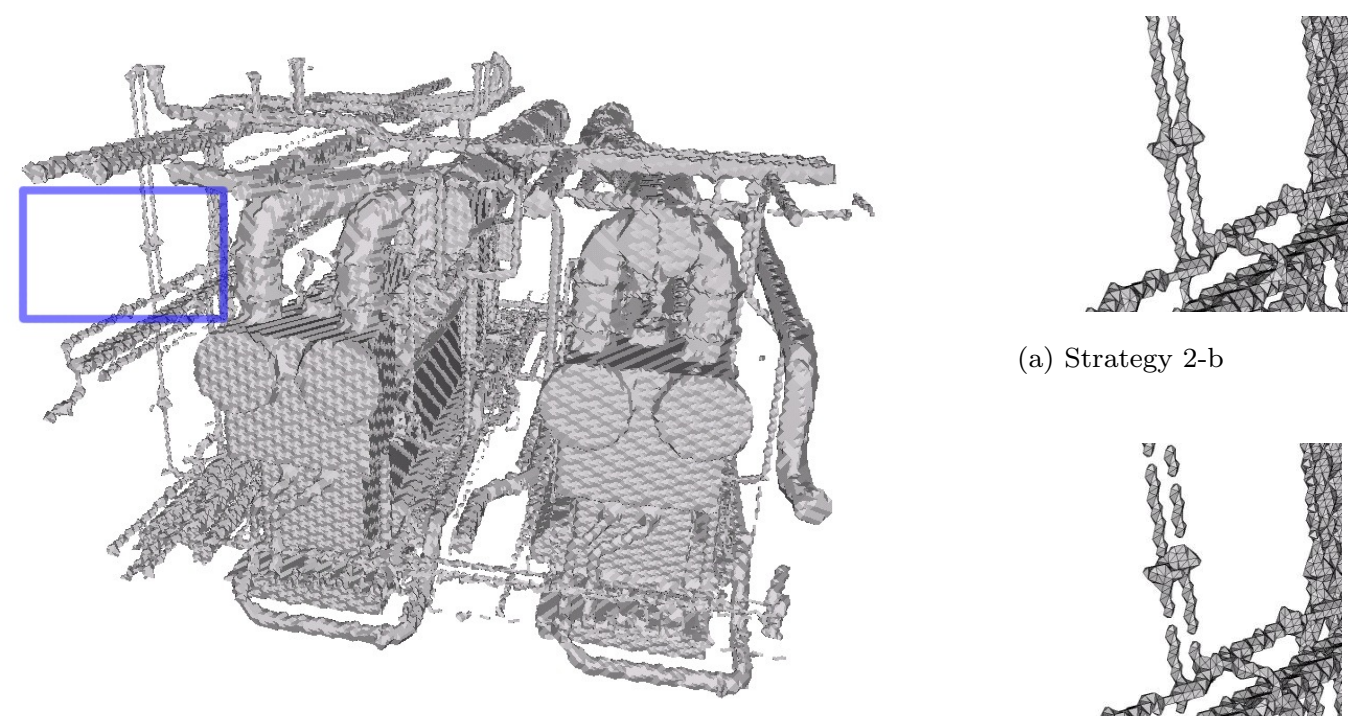

(a) Strategy 2-b

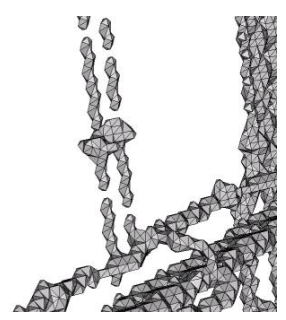

(b) Strategy 3-c

Figure 6: The result of two different strategies on a complex model. The right-hand side figures are blow-ups of the region marked with a blue rectangle

\section{Conclusions}

In this paper, four algorithms to control and to optimize the topological properties of isosurfaces have been presented and discussed. Several optimality criteria have been considered: total triangle count, genus, number of shells and combinations of these criteria. The remaining degrees of freedom in isosurface extraction algorithms have been identified, two data structures (the X-face graph and the Merge Tree of equivalence classes) that retain global topological properties of the final mesh $\mathrm{M}$ have been proposed, and several efficient algorithms (based on a few atomic operations and on the traversal of the X-face graph) for the topological control and optimization of the final triangular mesh have been presented and discussed.

Future work will focus on a deeper characterization of the algorithms based on the presented atomic decisions and on the development of area and volume minimization algorithms. Another topic for future work is how to use the proposed atomic decisions for reducing the total number of triangles counting once each group of adjacent, coplanar triangles. A systematic handling of ties when applying the atomic criteria is also an attractive goal.

\section{Acknowledgements}

The authors would like to thank Iordace Costin for his help. This work has been partially supported by the CICYT agency, under grant TIC-2001-2226-C01 and by a DARPA/NSF CARGO grant number 0138420. The original model on which the example of Figure 6 is based, was supplied by "Sener, Ingeniería y Sistemas".

\section{References}

[1] C. Andújar, P. Brunet, A. Chica, I. Navazo, J. Rossignac, and À. Vinacua. Computing maximal tiles and application to inpostor-based simplification. Computer Graphics Forum, 23(3), 2004. Proceedings of Eurographics'04.

[2] S. Bischoff and L. Kobbelt. Isosurface reconstruction with topology control. In Proc. Pacific Graphics 2002, pages 246-255, 2002.

[3] J. Bloomenthal. An implicit surface polygonizer. In Paul S. Heckbert, editor, Graphics Gems IV, pages 324-349. Academic Press, 1994. 


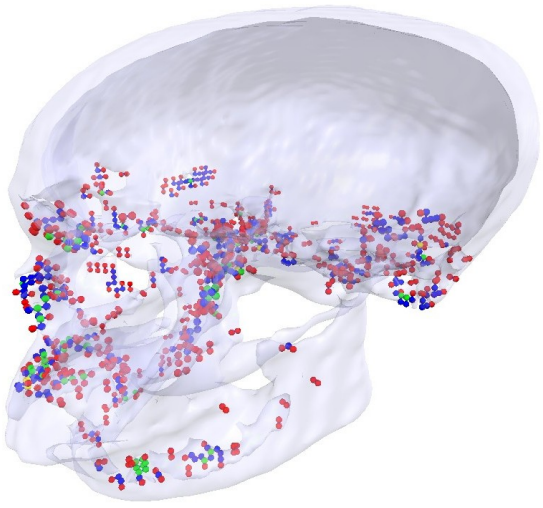

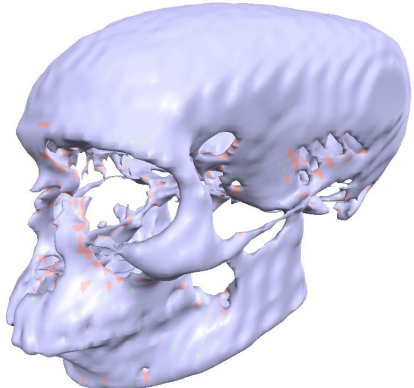

(b) Strategy 1-a

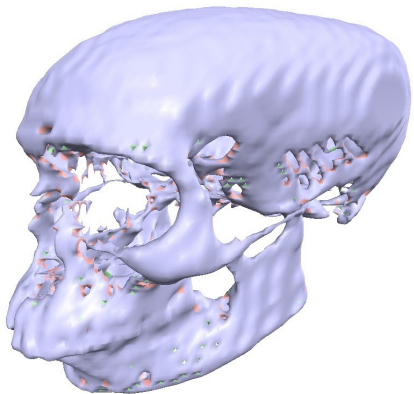

(d) Strategy 3-c

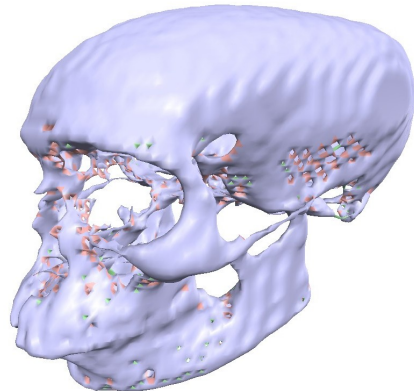

(c) Strategy 2-b

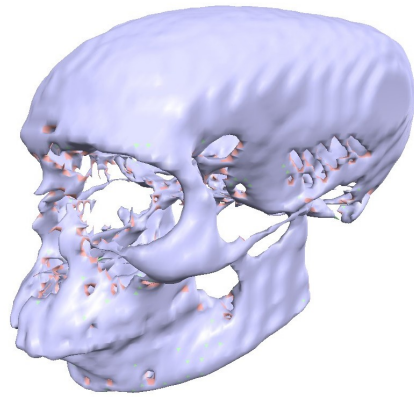

(e) Strategy 4-d

Figure 7: A model of a skull extracted from a CT scan with the four different strategies. The semi-transparent render on the left shows the X-face graph, with colors encoding the number of X-faces in each cube (red for one X-face, blue for two and green for three). In the four pictures on the right hand side, the pink triangles belong to cubes that have at least one X-face. The green triangles belong to X-cubes.

[4] P. Cignoni, F. Ganovelli, C. Montani, and R. Scopigno. Reconstruction of topologically correct and adaptive trilinear isosurfaces. Computers and Graphics, 24(3):399-418, 2000.

[5] M. J. Dürst. Letters: Additional reference to marching cubes. Computer Graphics, 22(2):72-73, 1988.

[6] I. Guskov and Z. Wood. Topological noise removal. In Proc. Graphics Interface 2001, Canada, pages 19-26, 2001.

[7] S. Hill and J. C. Roberts. Surface models and the resolution of n-dimensional cell ambiguity. In A. W. Paeth, editor, Graphics Gems V, pages 98-106. Academic Press, 1995.

[8] J.-O. Lachaud. Topologically defined iso-surfaces. In Proc. 6th Discrete Geometry for Computer Imagery (DGCI'96), Lyon, France, pages 245-256. Springer-Verlag, Berlin, 1996.

[9] W. Lorensen and H. Cline. Marching cubes: A high resolution 3D surface construction algorithm. Computer Graphics, 21(4):163-169, 1987.

[10] C. Montani, R. Scateni, and R. Scopigno. A modified look-up table for implicit disambiguation of marching cubes. The Visual Computer, 10(6):353-355, 1994.

[11] G. Nielson. On marching cubes. IEEE Transactions on Visualization and Computer Graphics, 9(3):283$297,2003$.

[12] G.M. Nielson, T.A. Foley, B. Hamann, and D. Lane. Visualizing and modeling scattered multivariate data. IEEE Computer Graphics and Applications, 11(3):47-55, 1991. 
[13] G.M. Nielson and B. Hamann. The asymptotic decider : Resolving the ambiguity in marching cubes. In Proc. of IEEE Visualization 91, pages 83-91, 1991.

[14] P. Ning and J. Bloomenthal. An evaluation of implicit surface tilers. IEEE Computer Graphics and Applications, 13(6):33-41, 1993.

[15] B. T. Stander and J. C. Hart. Guaranteeing the topology of an implicit surface polygonization for interactive modeling. Computer Graphics (SIGGRAPH 97 Proceedings), 31(1):279-286, 1997.

[16] A. Wallin. Constructing isosurfaces from ct data. IEEE Computer Graphics and Applications, 11(6):2833, 1991.

[17] J. Wilhelms and A. Van Gelder. Topological considerations in isosurface generation. Computer Graphics, 24(5):79-86, 1990.

[18] G. Wyvill, C. McPheeters, and B. Wyvill. Data structures for soft objects. The Visual Computer, 2(4):227-234, 1986.

[19] C. Zahlten. Piecewise linear approximation of isovalued surfaces. In F. H. Post and A. J. S. Hin, editors, Advances in Scientific Visualization, pages 105-118. Springer-Verlag, 1992. 